\title{
Primary spinal epidural non-Hodgkin's diffuse large B-cell lymphoma: a case report
}

\author{
Nishan Pokhrel ${ }^{1}$, Rohit Prasad ${ }^{1}$, Sushil Paudel ${ }^{1}$, Dinesh Kafle $^{1}$, and Rohit Pokharel ${ }^{1}$ \\ ${ }^{1}$ Tribhuvan University Institute of Medicine
}

May 11, 2020

\begin{abstract}
A 24-year-old male presented with radiating back pain and progressive neurological deficit. MRI revealed spinal epidural tumor at the thoracolumbar junction. He underwent decompression and excisional biopsy. Histopathology and immunohistochemistry identified it as diffuse large B-cell lymphoma. He received chemotherapy and is asymptomatic at one-year follow-up.
\end{abstract}

\section{Key clinical message}

Rarer diseases like primary spinal epidural diffuse large B-cell lymphomas should also be considered as the differential in those patients who complain of back pain with rapid neurological deterioration in lower limbs.

\section{KEYWORDS}

epidural, excisional biopsy, decompression, diffuse large B-cell lymphoma, non-Hodgkin's lymphoma, primary spinal epidural lymphoma

\section{Introduction}

Primary spinal epidural lymphomas (PSELs) comprise a group of tumors, which are present only in the spinal epidural space, with a histopathological picture of lymphoma, and negative diagnostic workup for lymphoma at other sites. ${ }^{1}$ Epidural location of lymphomas, both Hodgkin's and non-Hodgkin's is very rare due to which the diagnosis is challenging. About 24-48\% of non-Hodgkin's lymphomas (NHLs) were found to have extranodal origin. ${ }^{2,3}$ However, PSEL accounts for only $0.9 \%$ of all extranodal NHLs. ${ }^{4}$ There are many cell types of NHLs, DLBCL is the most common form which accounts for only $1.8 \%$ of all (nodal and extranodal) DLBCLs. ${ }^{5}$ It is easily missed and maybe misdiagnosed leading to lack of timely intervention and unhindered progression of the tumor.

This report describes the clinical features, imaging characteristics, and histopathological features of the rare case of primary spinal epidural diffuse large B-cell lymphoma (DLBCL). To the best of our knowledge, this is the first reported case of primary spinal epidural DLBCL from Nepal. It also emphasizes the importance of a multidisciplinary approach for the successful treatment of this disease.

\section{Case report}

A 24-year-old male came to seek medical attention for three months history of low back pain associated with the burning sensation of bilateral lower limbs. For the last 12 days, he was unable to walk due to gradually progressive weakness in bilateral lower limbs. These symptoms were not accompanied by fever, headache, 
and night sweats. He could not recall any history of trauma in the past. His past medical and surgical history was not significant.

On examination, there was a localized tenderness over the thoracolumbar region. Lower limb motor power varied across muscle groups: hip flexors $(2 / 5$ bilaterally), knee extensors $(2 / 5$ bilaterally $)$, ankle dorsiflexors ( $2 / 5$ bilaterally), long toe extensors ( $3 / 5$ bilaterally), and ankle plantar flexors ( $3 / 5$ bilaterally). The sensation of lower limbs was altered bilaterally, but bowel and bladder habits were normal. Deep tendon reflexes in the knee and the ankle joints were absent. Plantar reflexes were downgoing bilaterally.

With the clinical diagnosis of a space-occupying lesion in the thoracolumbar spine region, an X-ray of dorsolumbar spine was done. Apart from the loss of lumbar lordosis, the rest of the X-ray findings were normal. Magnetic resonance imaging (MRI) of the thoracolumbar spine with screening of the whole spine revealed extradural lesion extending from T10 to L2 vertebral level. T1W image showed homogenous lesion which was iso to hypo-intense and the $\mathrm{T} 2 \mathrm{~W}$ image showed heterogeneous lesion which was iso to hyperintense, compressing over the spinal cord dorsally (Figure 1,2). Sagittal short tau inversion recovery (STIR) image also revealed an extra-medullary lesion with high signal intensity to the vertebral marrow (Figure 3). Chest X-ray, complete blood count (CBC), liver and renal function tests, erythrocyte sedimentation rate (ESR), c-reactive protein (CRP), peripheral blood smear, lactate dehydrogenase (LDH), uric acid and urinalysis were normal. The diagnosis of the extradural tumor over the T10-L2 vertebrae was made.

Decompression (laminectomy) and excision biopsy of the mass was planned. The thoracolumbar spine (T9 to L3) was approached midline posteriorly. After laminectomy, a dorsally located greyish red extradural tumor extending from T10 to L2 was removed in strips. One of the long strips of the excised mass (approximately 15 $\mathrm{x} 1 \mathrm{~cm}$ ) was sent for histopathological examination (Figure 4). Histopathology identified it as DLBCL (Figure 5). Immunohistochemistry analysis showed CD3- ${ }^{-}$CD20 ${ }^{+}, \mathrm{CD}^{+}{ }^{+}, \mathrm{CD} 10^{+}, \mathrm{ALK}-1^{-}, \mathrm{BCL}-6^{+}, \mathrm{MUM}-1^{-}$and Ki- $67^{+}$. No other lesion was detected on staging evaluation which included contrast-enhanced computed tomography (CECT) of chest, abdomen, and pelvis, and bone marrow aspiration and biopsy.

His postoperative period was uneventful with successful recovery in his symptoms including neurology. He was referred to a cancer center after 16 days of hospital stay where he obtained six cycles of chemotherapy with rituximab, cyclophosphamide, doxorubicin, vincristine, and prednisolone (R-CHOP) regimen. After the treatment, the tumor regressed and he fully regained his muscle power. At the latest, one-year follow-up, he is asymptomatic and is performing his routine daily activity.

\section{Discussion}

Lymphomas are malignant tumors arising from lymphoid tissue. It can be Hodgkin's or non-Hodgkin's type, later being more common, and their primary epidural occurrence is very rare. ${ }^{4}$ Out of all the primary central nervous system (CNS) tumors (which also includes spinal lymphomas), primary DLBCL of the CNS constitutes only $0.5 \%$ cases, occurring in approximately 2 cases per 10 million people. Spinal cord location is particularly rare. ${ }^{6}$

The pathogenesis of primary central nervous system lymphomas (PCNSLs) has been a matter of considerable debate among experts. Some authors mention that PCNSL arise de novo in nervous system tissues due to the presence of lymphoid precursor cells in this location. ${ }^{7,8}$ Others argue that tumors from local and distant sites spread in the epidural space resulting in their apparent primary presentation. ${ }^{9}$ The remaining, believe that an unrecognized preexisting retroperitoneal disease may exist before they present as PSEL. ${ }^{10}$

There is a male preponderance (69\% vs $31 \%$ ) of PSEL matching exactly with our case. ${ }^{11}$ Though the usual age of presentation is beyond 40 years of age, several cases have been reported in younger patients. Nambiar et $\mathrm{al}^{12}$ reported a case of primary thoracic (T5-T10) spinal epidural B-lymphoblastic lymphoma in a 19year-old male from India. There are very few reported cases of spinal epidural NHL in younger age groups. ${ }^{13}$ Our case of a 24-year-old male is another addition to it. 
The thoracic spine is the most commonly involved site followed by the lumbosacral and cervical spine. ${ }^{8}$ The common occurrence of PSEL in the thoracic spine can be explained by the rich venous plexus in this site, greater length of the thoracic spine as compared with cervical and lumbar, more tolerance for bulky disease in the thorax and abdomen and the existence of unrecognized preexisting retroperitoneal disease. ${ }^{10,14}$ The usual location of the tumor in spinal epidural space is usually dorsal. ${ }^{15}$ Our case too had a similar finding of lesion present dorsally and located at the thoracolumbar region.

The onset of symptoms is usually subacute, occurring over a few days to weeks. The symptoms and signs of the disease depend on the location and size of the tumor. Localized back pain followed by lower limb weakness is the most common symptom. ${ }^{11}$ Local back pain, sometimes accompanied by radicular pain to legs and abdomen can be the prodromal symptom that can persist for several months to a year. Then, the phase of rapid neurological deterioration occurs over 2-8 weeks which is due to spinal cord compression. ${ }^{14}$ Bladder and bowel disturbances appear only later in the course. ${ }^{16}$ It may give us the idea of time since the onset of the pathology. The presenting features in our case were back pain radiating to bilateral lower limbs for three months followed by neurological deficits for the last 12 days without bladder involvement.

B symptoms (fever, night sweats, and weight loss) were found to be rare and none had hepatomegaly or splenomegaly in one of the largest surveys, which is consistent with our case too. As most of the patients presented in the earlier stage, B symptoms were probably absent. ${ }^{11}$

MRI is the investigation of choice in the preoperative and postoperative evaluations. ${ }^{10}$ PSEL appears in MRI as isointense on T1W image and iso- to hyperintense on T2W image, with marked contrast enhancement. ${ }^{15}$ Contrast enhancement with Gadolinium further helps in the assessment of extraosseous soft tissue components. This allows a better differentiation of various pathologies (e.g., metastatic carcinoma and sarcoma), tumor extent, and bony involvement. ${ }^{15}$ Similar features were recognized in our case too.

The whole-body scan should be performed to identify lungs, pleural, splenic, gastric, intestinal, pancreatic, and renal involvement. Bone marrow biopsy from the sternum or iliac crest is required to rule out lymphoreticular involvement. CBC, LDH, and chest X-ray should be performed as well. In our case, we could not find any positive reports. Lumbar puncture is not recommended because it is potentially hazardous because of the coning phenomenon and it is also of limited diagnostic value as it shows a non-specific increase in protein levels; neoplastic cells are rarely found. ${ }^{17,18}$

Monnard et $\mathrm{al}^{11}$ have recommended surgical decompression in the form of partial or total removal of the tumor mass and/or decompressive laminectomy. Doing so relieves the spinal cord compression and also establishes the correct histological diagnosis. Surgery is clearly indicated when the diagnosis is not yet established. ${ }^{1}$ Emergency decompression is needed only in cases of acute paresis and/or loss of bowel/bladder control. ${ }^{16}$ Functional recovery of patients suffering spinal cord compression due to PSEL is relatively better than that of patients with metastatic carcinoma. ${ }^{4}$

Histopathological examination of the biopsy specimen usually shows diffuse proliferation of atypical lymphoid cells ${ }^{8}$ which is similar to our finding. Immunohistochemistry analysis with positive CD20 ${ }^{+}, \mathrm{CD} 10^{+}, \mathrm{BCL} 6^{+}$, and MUM-1- $1^{-}$further supported our diagnosis of DLBCL (germinal center type). ${ }^{5,}{ }^{19}$ Positive Ki-67 suggested the aggressiveness of the tumor.

Combined modality treatment including radiotherapy and chemotherapy has been recommended to be the most efficient treatment because lymphomas are very chemo- and radiosensitive tumors. ${ }^{11,}{ }^{18,}{ }^{20}$ In the largest survey until now, local control of $88 \%$ and 5 -year overall survival of $69 \%$ have been observed with combined modality treatment. ${ }^{11}$ Earlier diagnosis and treatment is associated with improved functional outcomes. Several authors report that patients with more aggressive histological tumor types have a poorer prognosis. Patients of over 40 years of age with aggressive histological tumors have a poor prognosis. ${ }^{10}$ Our case was a 24 year-old male, otherwise healthy, and was diagnosed early and underwent surgery with significant neurological improvement, which is the most favorable prognostic indicator of overall survival. ${ }^{11}$ He responded well to chemotherapy with complete neurological recovery. 


\title{
Conclusion
}

Primary spinal epidural DLBCL is one of the rarest tumors due to which its diagnosis is challenging. Signs and symptoms are similar to those of any other epidural space-occupying lesions and thus, can be frequently misdiagnosed. Back pain with sudden neurological deterioration in lower limbs should raise the suspicion of rarer diseases like primary spinal epidural DLBCL. A high degree of suspicion along with team approach (surgeons, radiotherapist, oncologist, and pathologist) is vital for its diagnosis and management.

\author{
List of Abbreviations \\ $\mathrm{CBC}=$ Complete Blood Count \\ $\mathrm{CECT}=$ Contrast Enhanced Computed Tomography \\ $\mathrm{CNS}=$ Central Nervous System \\ DLBCL $=$ Diffuse large B-cell lymphoma \\ $\mathrm{LDH}=$ Lactate Dehydrogenase \\ MRI = Magnetic Resonance Imaging \\ NHL $=$ Non-Hodgkin's Lymphoma \\ PCNSL $=$ Primary Central Nervous System Lymphoma \\ PSEL = Primary Spinal Epidural Lymphoma \\ R-CHOP = Rituximab, Cyclophosphamide, Doxorubicin, Vincristine and Prednisolone, \\ STIR $=$ Short Tau Inversion Recovery \\ $\mathrm{T} 1 \mathrm{~W}=\mathrm{T} 1$ Weighted \\ $\mathrm{T} 2 \mathrm{~W}=\mathrm{T} 2$ Weighted
}

\section{Declaration}

Ethics approval and consent to participateNeed for ethical approval waived. Consent from the patients' party deemed to be enough.Consent for publicationWritten informed consent were obtained from the patient for publication of this case report and any accompanying images. A copy of the written consent is available for review by the editor-in-chief of this journal.Availability of data and materialNot applicableConflict of interestThe authors declare that there is no conflict of interest regarding the publication of this paper.FundingNoneAuthor contributionsNBP and RP: wrote the initial draft, edited it and reshaped into this manuscript. RKP reviewed the manuscript. All authors approved the final version of the manuscript and agreed to be accountable for all aspects of the work in ensuring that questions related to the accuracy or integrity of any part of the work are appropriately investigated and resolved.AcknowledgmentsThe authors are particularly grateful to Dr. Pradeep Raj Regmi, Pediatric Imaging European School of Radiology (ESOR), University Hospital of Ioannina, Greece for his valuable insights of the radiological images; Dr. Nisha Sharma, Department of Pathology, Tribhuvan University Institute of Medicine, Kathmandu, Nepal for her inputs on histopathological slides. We are also thankful to the patient and his family for their valuable support in the preparation of this manuscript.

\section{References}

1. Cugati G, Singh M, Pande A, et al. Primary spinal epidural lymphomas.J Craniovertebr Junction Spine . 2011, 2(1):3. https://doi.org/10.4103/0974-8237.85307

2. Freeman C, Berg JW, Cutler SJ. Occurrence and prognosis of extranodal lymphomas: Cancer . 1972, 29(1):252-60. URL: https://pubmed.ncbi.nlm.nih.gov/5007387/ 
3. Otter R, Gerrits WB, vd Sandt MM, Hermans J, Willemze R. Primary extranodal and nodal nonHodgkin's lymphoma: A survey of a population-based registry. Eur J Cancer Clin Oncol . 1989, 25(8):120310. https://doi.org/10.1016/0277-5379(89)90416-1

4. Tsukada T, Ohno T, Tsuji K, et al. Primary epidural non-Hodgkin's lymphoma in clinical stage IEA presenting with paraplegia and showing complete recovery after combination therapy. Intern Med . 1992, 31(4):513-5. https://doi.org/10.2169/internalmedicine.31.513

5. Wada N, Kohara M, Ikeda J, et al. Diffuse large B-cell lymphoma in the spinal epidural space: A study of the Osaka Lymphoma Study Group.Pathol Res Pract . 2010, 206(7):439-44. https://doi.org/10.1016/j.prp.2010.02.003

6. Páscoa Pinheiro J, Rato J, Rebelo O, Costa G. Primary spinal epidural lymphoma: a rare entity with an ambiguous management. BMJ Case Rep . 2020, 13(1):e233442. Accessed: April 15, 2020: https://doi.org/10.1136/bcr-2019-233442

7. Morgello S, Lagoo AS. Nervous system involvement by lymphoma, leukemia, and other hematopoietic cell proliferations. In McLendon RE, Rosenblum MK, Binger DD, ed. Russell and Rubinstein's Pathology of Tumors of the Nervous System . 7, Euston Road, London: Hodder Arnold; 2006:837-902. https://doi.org/10.1201/b13439

8. Xiong L, Liao LM, Ding JW, Zhang ZL, Liu AW, Huang L. Clinicopathologic characteristics and prognostic factors for primary spinal epidural lymphoma: report on 36 Chinese patients and review of the literature. BMC cancer . 2017, 17(1):131. https://doi.org/10.1186/s12885-017-3093-z

9. Grant JW, Kaech D, Jones DB. Spinal cord compression as the first presentation of lymphoma-a review of 15 cases. Histopathology . 1986, 10(11):1191-202. https://doi.org/10.1111/j.1365-2559.1986.tb02559.x

10. Lyons MK, O'Neill BP, Marsh WR, Kurtin PJ. Primary spinal epidural non-Hodgkin's lymphoma: report of eight patients and review of the literature. Neurosurgery . 1992, 30(5):675-80. https://doi.org/10.1227/00006123-199205000-00004

11. Monnard V, Sun A, Epelbaum R, et al. Primary spinal epidural lymphoma: patients' profile, outcome, and prognostic factors: a multicenter Rare Cancer Network study. Int J Radiat Oncol Biol Phys . 2006, 65(3):817-23. https://doi.org/10.1016/j.ijrobp.2006.01.002

12. Nambiar RK, Nair SG, Prabhakaran PK, Mathew SP. Primary spinal epidural B-lymphoblastic lymphoma. Proc (Bayl Univ Med Cent) . 2017, 30(1):66-68. https://doi.org/10.1080/08998280.2017.11929533

13. Mally R, Sharma M, Khan S, Velho V. Primary lumbo-sacral Spinal epidural non-Hodgkin's lymphoma: A case report and review of literature.Asian Spine $J$. 2011, 5(3):192-195. https://doi.org/10.4184/asj.2011.5.3.192

14. Epelbaum R, Haim N, Ben-Shahar M, Ben-Arie Y, Feinsod M, Cohen Y. Non-Hodgkin's lymphoma presenting with spinal epidural involvement.Cancer . 1986, 58(9):2120-4. https://doi.org/10.1002/10970142(19861101)58:9<2120::AID-CNCR2820580926>3.0.CO;2-A

15. Boukobza M, Mazel C, Touboul E. Primary vertebral and spinal epidural non-Hodgkin's lymphoma with spinal cord compression.Neuroradiology . 1996, 38(4):333-7. https://doi.org/10.1007/BF00596582

16. Hong B, Hermann EJ, Reuter C, Brandis A, Krauss JK. Outcome of surgical decompression of spinal mass lesions in non-Hodgkin's lymphoma and plasmacytoma. Clin Neurol Neurosurg . 2013, 115(12):247681. https://doi.org/10.1016/j.clineuro.2013.09.037

17. Spinazze S, Caraceni A, Schrijvers D. Epidural spinal cord compression. Crit Rev Oncol Hematol. 2005, 56(3):397-406. https://doi.org/10.1016/j.critrevonc.2005.04.005 
18. Haddad P, Thaell JF, Kiely JM, Harrison EG, Miller RH. Lymphoma of the spinal extradural space. Cancer . 1976, 38(4):1862-6. https://doi.org/10.1002/1097-0142(197610)38:4<1862::AIDCNCR2820380467>3.0.CO;2-8

19. Hans CP, Weisenburger DD, Greiner TC, et al. Confirmation of the molecular classification of diffuse large B-cell lymphoma by immunohistochemistry using a tissue microarray. Blood . 2004, 103(1):275-82. https://doi.org/10.1182/blood-2003-05-1545

20. Barnard M, Perez-Ordonez B, Rowed DW, Ang LC. Primary spinal epidural mantle cell lymphoma: case report. Neurosurgery . 2000, 47(5):1239-42. https://doi.org/10.1097/00006123-200011000-00046

\section{Figure captions}

Figure 1: Sagittal T1W image shows well-defined iso to hypointense lesion extending from the lower level of T10 vertebral body to L2 vertebral level in extramedullary region (red arrow). The lesion is severely compressing the spinal cord. The vertebral bodies show normal signal intensity. Intervertebral disc space is well maintained and no prevertebral collection can be appreciated. T1W, T1-weighted

Figure 2: Sagittal (A) and axial (B) T2W images show well-defined, heterogeneous, extra-medullary, iso to hyperintense lesion (red arrow) extending from T10 to L2 vertebral levels, compressing and displacing the spinal cord anteriorly. No obvious erosion of the vertebral body is seen. T2W, T2-weighted

Figure 3: Sagittal STIR image showing extra-medullary lesion (red arrow) extending from T10-L2 levels with high signal intensity to the vertebral marrow which is compressing and displacing the spinal cord anteriorly. STIR, short tau inversion recovery

Figure 4: A $15 \times 1 \mathrm{~cm}$ grey to white-colored lesion which was soft to firm in consistency

Figure 5: Histopathology (hematoxylin and eosin, x 200) revealing diffuse proliferation of intermediate-sized atypical lymphoid cells infiltrating into the fibrocollagenous tissue. Few of the cells show nuclear irregularity with prominent nucleoli. Small lymphocytes are also seen in the adjacent area. 


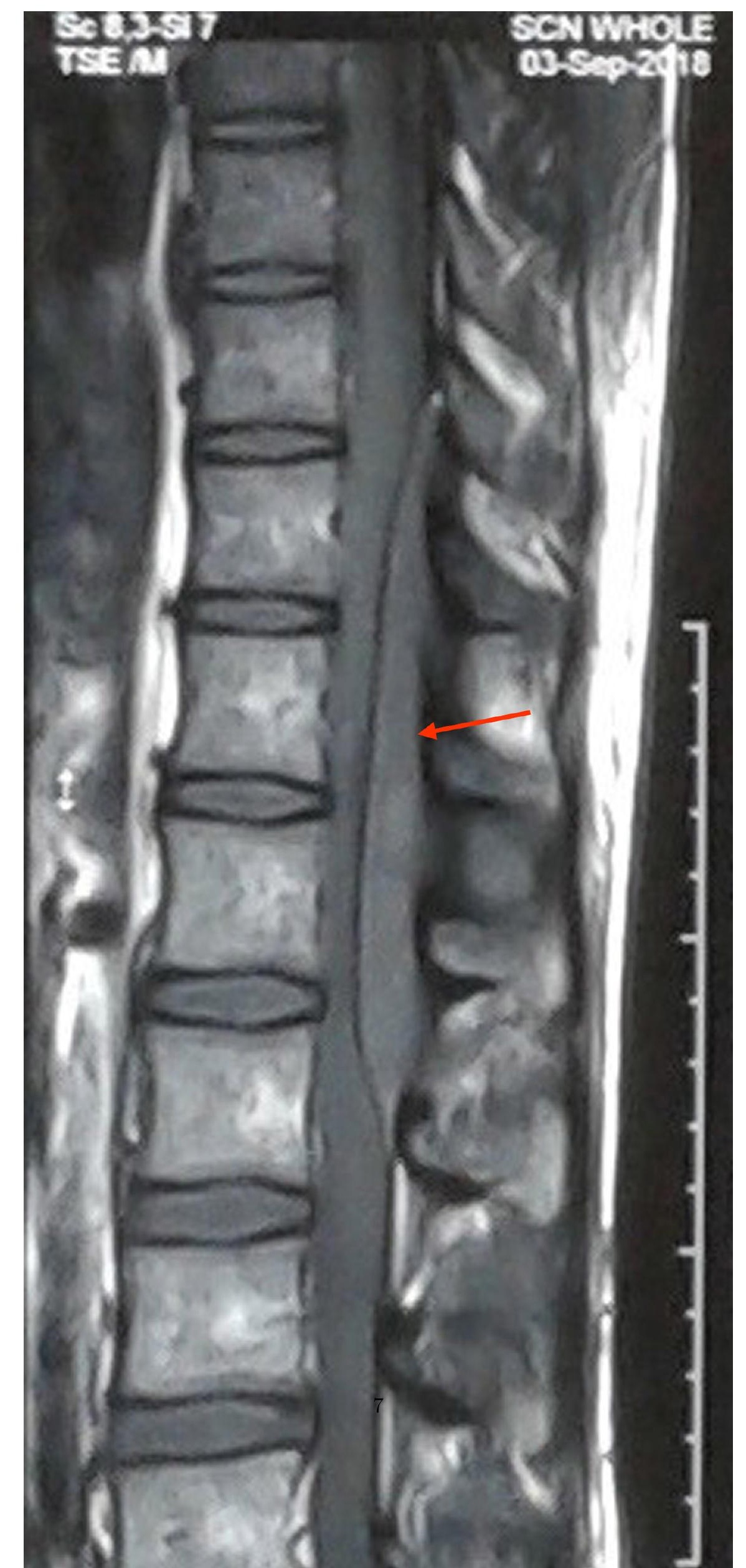




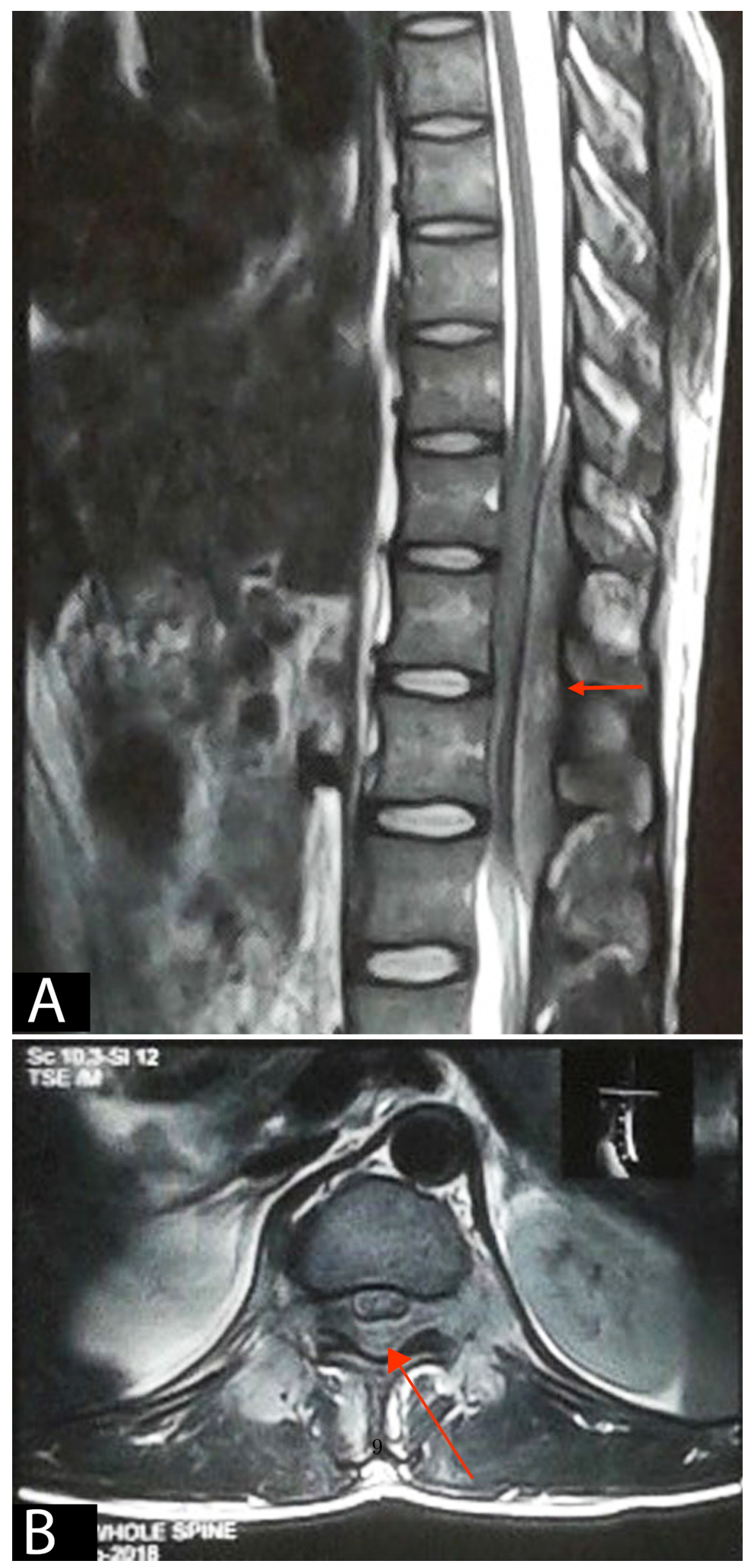




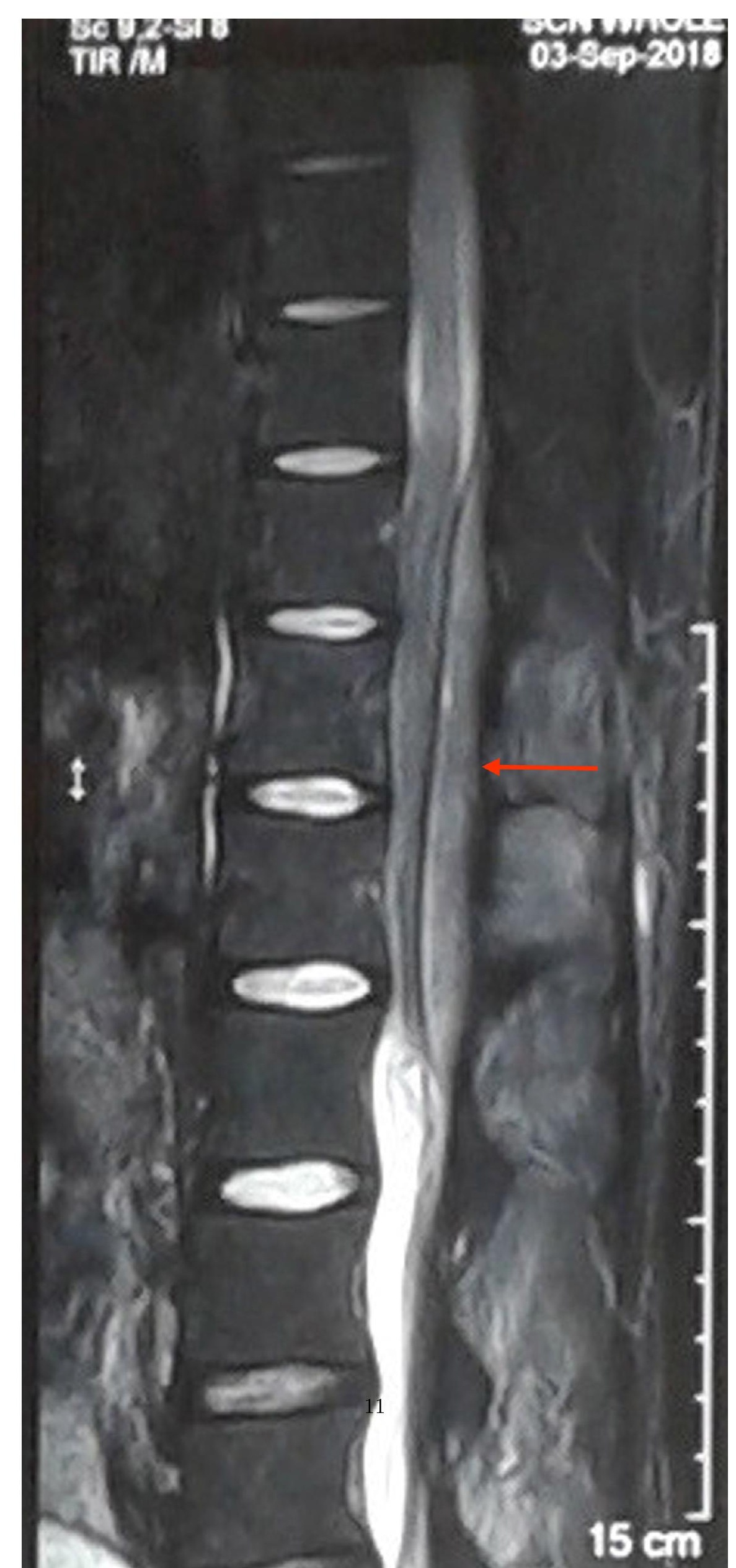




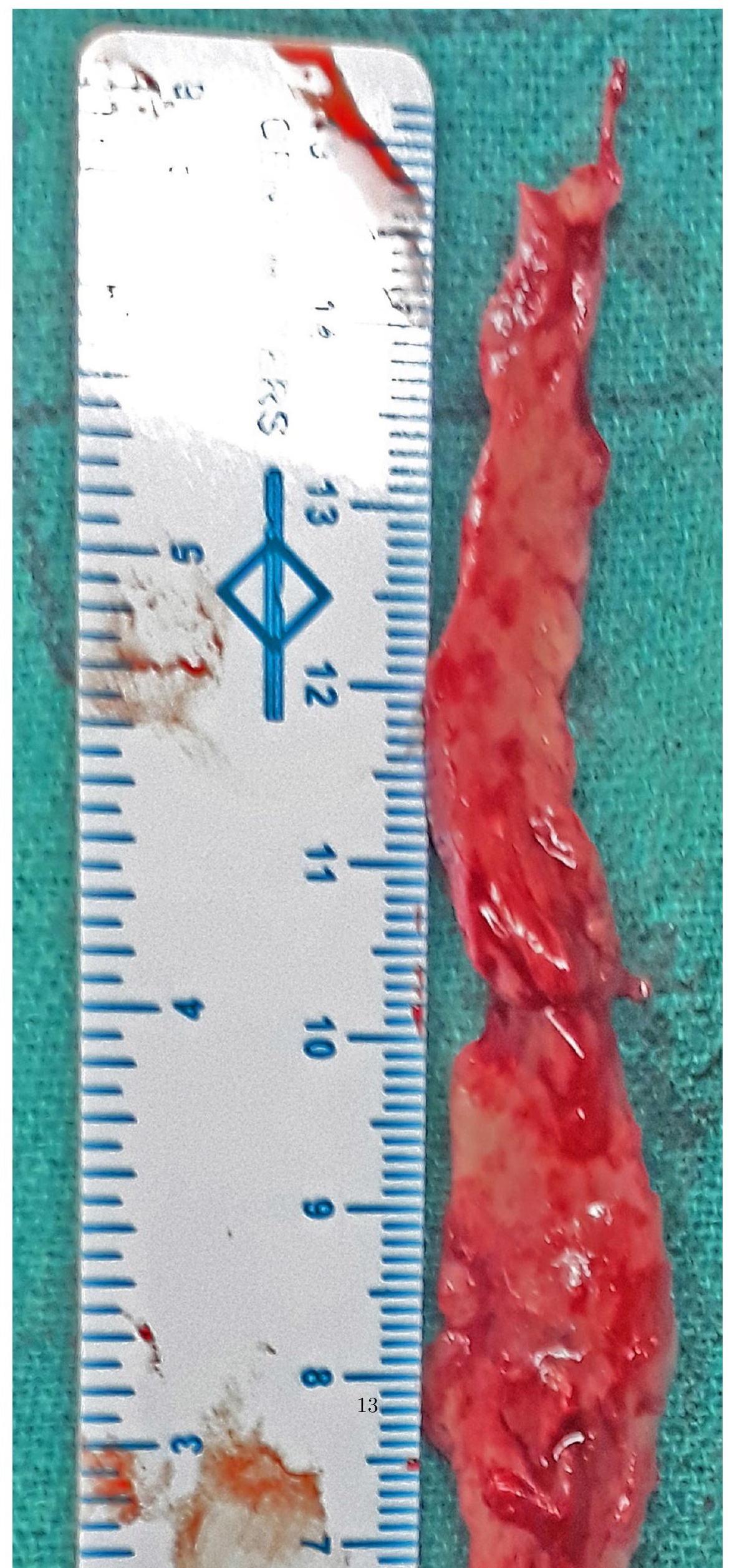




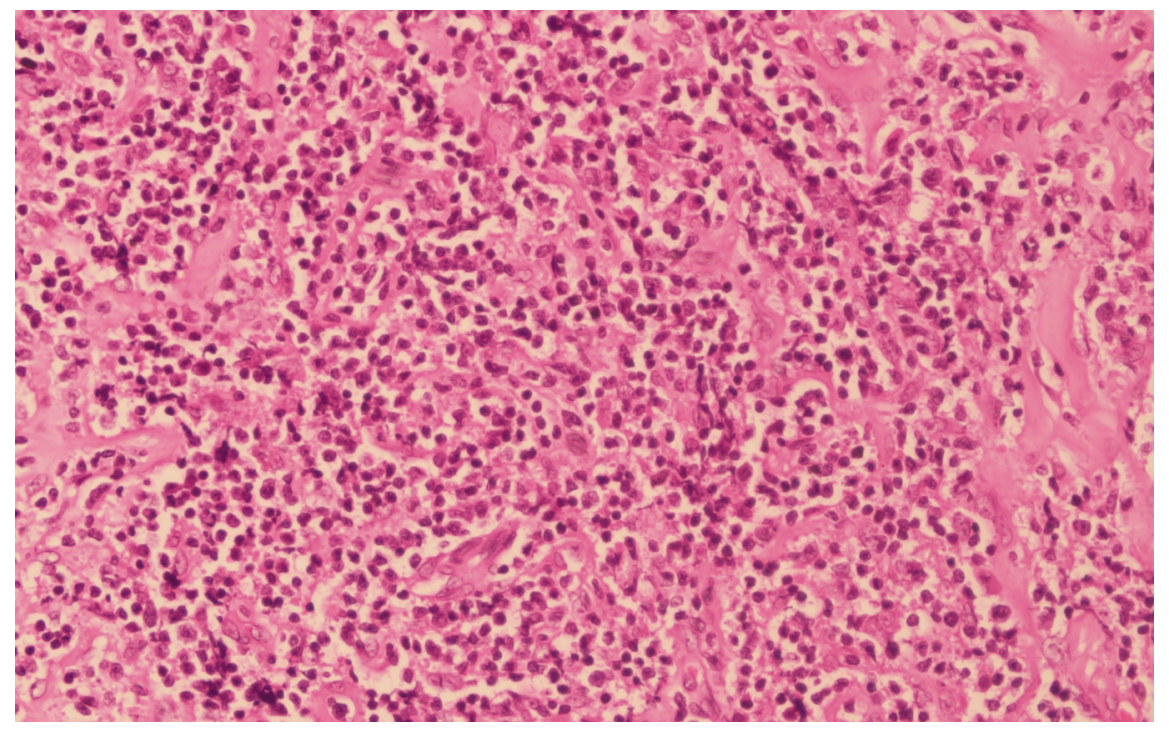

